\title{
Cirugía mínimamente invasiva del cáncer gástrico
}

\author{
Carlos García C. ${ }^{1,2}$ y Lorenzo González P. ${ }^{1}$
}

\section{Minimally invasive surgery for gastric cancer}

Standard surgery for gastric cancer has been defined in recent decades by evidence mainly from the East, where updates for minimally invasive management have been incorporated, especially in the early stages of this disease. There is current evidence from multiple randomized and controlled studies comparing minimally invasive surgery and open surgery in gastric cancer. Consequently, we can affirm with sufficient support that in early gastric cancer, laparoscopic distal gastrectomy can be considered as the standard treatment. However, more results should be expected to make the same statement for total gastrectomies, both for early and locally advanced gastric cancer. The aim in this update is to report on the current available evidence in the management of gastric cancer with minimally invasive treatment.

Key words: minimally invasive surgery; laparoscopy; stomach neoplasm; gastrectomy.

\section{Resumen}

La cirugía estándar del cáncer gástrico ha sido definida en las últimas décadas por evidencia principalmente entregada desde oriente, donde se han incorporado actualizaciones para el manejo mínimamente invasivo, especialmente en estadios iniciales de esta enfermedad. Existe evidencia actual, entregada por múltiples estudios randomizados y controlados, que comparan la cirugía mínimamente invasiva y cirugía abierta en cáncer gástrico. Es así que podemos afirmar con suficiente respaldo, que en cáncer gástrico incipiente la gastrectomía subtotal laparoscópica se puede considerar como el tratamiento estándar. Sin embargo, aún se deben esperar más resultados para aseverar lo mismo en el caso de las gastrectomías totales, tanto para cáncer incipiente como avanzado. Nuestro objetivo en esta actualización es incluir la evidencia actual disponible en el manejo del cáncer gástrico en relación al tratamiento mínimamente invasivo.

Palabras clave: cirugía mínimamente invasiva; laparoscopía; cáncer gástrico; gastrectomía.

\section{Introducción}

A nivel mundial, el cáncer gástrico (CG) ocupa el tercer lugar como causa de muerte por enfermedades neoplásicas ${ }^{1}$. Es especialmente frecuente en países del este asiático, Europa Central y algunos de la costa pacífica de América Latina. Por el contrario, es poco frecuente en Europa Occidental y Norteamérica $^{2,3}$. En Chile, el cáncer gástrico ocupa el primer lugar como causa de muerte por neoplasias y conlleva una fuerte carga de enfermedad para nuestro sistema de salud ${ }^{4}$.

Existe una tendencia global a disminución de las tasas de incidencia y mortalidad, a pesar que algunos tipos específicos han mostrado una inclinación contraria, como es el caso de los tumores poco $\operatorname{cohesivos}^{5} \mathrm{y}$ los tumores de localización más alta ${ }^{6}$.

Dada la alta incidencia de esta neoplasia en países del este asiático, se han implementado pro- gramas de tamizaje o screening que han llevado a que países como Japón y Corea pesquisen una alta proporción de lesiones precoces, es decir, cáncer gástrico incipiente ${ }^{7,8}$. En este sentido, la rápida introducción de la cirugía mínimamente invasiva impulsó a muchos grupos de estos países a introducir la cirugía laparoscópica en el manejo de esta neoplasia9 . Es así como en 1994, Kitano realizó la primera gastrectomía distal laparoscópica en pacientes con cáncer gástrico incipiente ${ }^{10}$. Desde entonces, la gastrectomía laparoscópica ha ganado enorme y creciente popularidad, sin embargo, persisten algunas controversias principalmente enfocadas en $\mathrm{su}$ aplicación al cáncer gástrico avanzado ${ }^{11,12}$.

Los esfuerzos de muchos grupos, tanto orientales como occidentales, están enfocados en demostrar, a través de un buen nivel de evidencia, la no inferioridad de la cirugía laparoscópica con respecto a la cirugía abierta, en relación a morbilidad y
Servicio y Departamento de Cirugía, Hospital Clínico San Borja-Arriarán. Campus Centro, Facultad de Medicina, Universidad de Chile.

2Departamento de Cirugía Adultos, Clínica Las Condes.

Financiación: Sin apoyo financiero.

Recibido el 19 de junio de 2020, aceptado para publicación el 19 de junio de 2020.

Correspondencia a: Dr. Carlos García Carrasco dr.carlosgarciac@gmail.com 
mortalidad, así como en la sobrevida a largo plazo; añadiendo los beneficios de la cirugía mínimamente invasiva $^{13,14}$.

Desde hace tres décadas, la cirugía estándar del cáncer gástrico ha sido definida por evidencia recopilada principalmente en Japón y luego en Corea. Esta evidencia se ha traducido, entre otros documentos, en las "Guías Japonesas de Tratamiento del Cáncer Gástrico"15, las cuales se han ido actualizando periódicamente y han introducido, en las últimas ediciones, los conceptos de manejo mínimamente invasivo para los estadios más precoces de esta enfermedad. En la última edición de 2018, quedó establecido que la cirugía laparoscópica es una opción válida para el manejo del cáncer gástrico cT1 por medio de una gastrectomía subtotal distal. Sin embargo, también establece la precaución en cáncer gástrico avanzado y en la técnica de gastrectomía total, por la ausencia de evidencia suficiente para ser plasmada en una guía clínica ${ }^{16}$.

El objetivo de la presente comunicación es aportar, con la mejor evidencia disponible, el rol actual de la cirugía laparoscópica en el manejo del cáncer gástrico.

\section{Cirugía mínimamente invasiva en cáncer gástrico incipiente}

Por la alta incidencia y proporción de cáncer gástrico incipiente observada en Japón y Corea, no es de extrañar que la cirugía laparoscópica haya irrumpido tan definidamente en estos países y en este estadio de la enfermedad. Más del 50\% de los pacientes diagnosticados y tratados tienen lesiones incipientes, localizadas en el estómago distal y con una baja incidencia de diseminación linfática, condiciones ideales para realizar una gastrectomía laparoscópica ${ }^{17}$.

En cuanto a los objetivos y principios de la cirugía del cáncer gástrico (válido también para lesiones avanzadas) éstos pueden ser resumidos en: Resección gástrica R0, es decir, gastrectomía con bordes macroscópicos e histológicos negativos (pudiendo incluir resección de órganos vecinos); y disección linfática extendida y estandarizada. También es posible considerar como objetivos, una baja morbimortalidad, rápida recuperación quirúrgica y mínimo impacto en la calidad de vida. Pero evidentemente, el objetivo o fin último de la cirugía es intentar curar la enfermedad ${ }^{16}$ ¿Puede la cirugía laparoscópica en cáncer gástrico conseguir estos objetivos? ¿Cuál es la evidencia?

Hasta la fecha, se han publicado más de una decena de trabajos o estudios randomizados y controlados, que comparan la cirugía mínimamente invasiva versus la cirugía clásica en cáncer gástrico incipiente. El primero de éstos fue publicado por el propio Kitano, en una serie randomizada, con un número bajo de pacientes, y que comparó la gastrectomía distal laparoscópica versus la gastrectomía distal abierta en cáncer gástrico incipiente ${ }^{18}$. El método de reconstrucción posterior a la resección, fue la gastro-duodeno anastomosis o Billroth I, técnica empleada muy excepcionalmente en occidente. Este primer estudio, demostró que los pacientes sometidos a gastrectomía laparoscópica experimentaron una menor pérdida sanguínea, menos dolor postoperatorio, una recuperación más rápida $\mathrm{y}$, más importante aún, resultados oncológicos similares. Desde entonces, varios otros estudios randomizados, ahora a mayor escala, han demostrado que la gastrectomía distal laparoscópica es igual, sino superior a la gastrectomía distal abierta. El estudio más señero es el KLASS- $01^{19,20}$ realizado en Corea y que demostró la no inferioridad de la gastrectomía laparoscópica distal comparada con cirugía abierta en cáncer gástrico incipiente distal, tanto en resultados quirúrgicos precoces como en los alejados u oncológicos. Con posterioridad, el estudio JCOG0912 ${ }^{21}$ realizado en Japón, confirmó la equivalencia de la cirugía laparoscópica y abierta en resultados postoperatorios tanto precoces como alejados y, por lo tanto, debería ser considerado el estándar de tratamiento cuando es realizada por cirujanos de experiencia ${ }^{22}$.

El análisis más detallado del estudio coreano $\left(\right.$ KLASS-01) ${ }^{20}$ demostró, en el estudio de aproximadamente 1.400 pacientes (alrededor de 700 pacientes por rama), que la cirugía laparoscópica presentó un tiempo operatorio mayor, y que la cosecha ganglionar fue significativamente menor que en la cirugía abierta. Uno de los principales métodos para objetivar la calidad de la cirugía en cáncer gástrico, es el recuento de ganglios examinados por el patólogo. Si bien en ambos grupos se obtuvo una cosecha ganglionar considerada suficiente para una adecuada etapificación, este resultado podría tener implicancia si se hubiesen analizado pacientes con cáncer gástrico avanzado. Otro estudio interesante es el COACT 0301, que a pesar de tener resultados similares en el grupo de cirugía laparoscópica, no ve mayor beneficio de ésta a largo plazo ${ }^{23}$.

Estos resultados han sido corroborados en metaanálisis que han resumido los resultados obtenidos en estos estudios randomizados ${ }^{24-27}$. La conclusión de ellos es que la cirugía laparoscópica en gastrectomía distal es estadísticamente similar a la cirugía abierta en cuanto a resultados a corto y largo plazo. 
En occidente, la implementación de la cirugía laparoscópica para cáncer gástrico ha sido mucho más lenta en comparación a oriente ${ }^{28-30}$. La principal razón de este atraso se debe en parte a que en nuestros países la gran mayoría de los pacientes con este diagnóstico, debutan con tumores avanzados (compromiso transmural), diseminación linfática extensa o, definitivamente, con diseminación de distancia; lo que entorpece y desincentiva iniciar una experiencia segura en cirugía laparoscópica ${ }^{31}$.

La evidencia recopilada en occidente está basada principalmente en estudios no randomizados, y que llegan a la conclusión que en pacientes bien seleccionados, la gastrectomía laparoscópica es segura y efectiva como vía de abordaje para CG incipiente $\mathrm{t}^{32,33}$. Sólo ha sido publicado un estudio randomizado, con bajo número de pacientes con tumores incipientes $(20 \%)$, realizado por $\mathrm{Husher}^{34}$ en 2005 , que llega a las mismas conclusiones mencionadas.

A diferencia de la gastrectomía subtotal, no existe evidencia robusta ni definitiva en gastrectomía total para cáncer gástrico incipiente. En la actualidad, hay dos estudios randomizados que esperan la publicación de los resultados finales; CLASS02-01 y KLASS-03 sólo han publicado los protocolos de estudio, no así los resultados ni conclusiones fina$\operatorname{les}^{35,36}$. La gastrectomía total laparoscópica es un procedimiento complejo que requiere habilidades quirúrgicas laparoscópicas avanzadas, principalmente en la confección de la anastomosis esófagoyeyunal $^{37}$. La falta temporal de evidencia robusta no invalida su uso en grupos de experiencia ${ }^{38}$.

Hay en la actualidad, varios otros estudios ran- domizados que evalúan otros aspectos de la cirugía mínimamente invasiva en cáncer gástrico incipiente; evaluación de cirugía económica con estudio de linfonodo centinela (SENORITA) ${ }^{39}$, gastrectomía con preservación pilórica (KLASS-04) ${ }^{40}$, gastrectomía proximal laparoscópica (KLASS-05 y JCOG1401 $)^{41,42}$ y gastrectomía distal completamente laparoscópica (KLASS07) ${ }^{43}$, entre otros (Tabla 1).

\section{Cirugía mínimamente invasiva para cáncer gástrico avanzado}

A pesar de la extensa literatura que apoya el uso de la cirugía mínimamente invasiva en el cáncer gástrico incipiente, no existe hasta el momento la misma disponibilidad de evidencia para su aplicación en cáncer gástrico avanzado. Esto no implica, de ninguna manera, que la cirugía laparoscópica sea inferior o no esté indicada en estos tumores ${ }^{38}$. En la actualidad, muchos grupos quirúrgicos consideran la vía laparoscópica como el estándar de tratamiento del cáncer gástrico avanzado.

Las guías japonesas (Japanese Gastric Cancer Treatment Guidelines 2018 (5th edition)) y norteamericanas (National Comprehensive Cancer Network) indican textualmente: "...que no existe evidencia suficiente para recomendar esta vía de abordaje, ya que los estudios randomizados al respecto, aún no publican resultados finales o que esta vía requiere más investigación"16,17.

La cirugía mínimamente invasiva para cáncer gástrico requiere de habilidades quirúrgicas avanzadas. Las dificultades técnicas, diferentes a la

Tabla 1. Principales estudios randomizados para cáncer gástrico incipiente: cirugía laparoscópica versus abierta

\begin{tabular}{|c|c|c|c|c|c|c|}
\hline Estudio & País & Fase & Intervención & $\begin{array}{l}\text { Muestra } \\
\text { pacientes }\end{array}$ & $\begin{array}{l}\text { Criterios de } \\
\text { inclusion }\end{array}$ & “End-Point" \\
\hline KLASS- $01^{19,20}$ & Corea & III & GSTD lap $v s$ abierta & 1.400 & cT1-2, N0-1 & Sobrevida 5 años \\
\hline KLASS- $03^{36}$ & Corea & II & GT lap vs GT abierta & 168 & cT1 & Morbilidad, mortalidad \\
\hline SENORITA $^{39}$ & Corea & III & SSN vs G lap & 580 & cT1N0 & Sobrevida 3 años \\
\hline KLASS- $07^{43}$ & Corea & II & GSTD asistida lap vs GSTD lap total & 442 & Estadio IA/IB & Morbilidad, mortalidad \\
\hline СOACT0301 23 & Corea & III & GSTD lap $v s$ abierta & 164 & cT1N0-1 & Sobrevida 5 años \\
\hline CLASS02-0135 & China & II & GT lap vs GT abierta & 200 & cT1-2, cN0-1 & Morbilidad, mortalidad \\
\hline JCOG $1401^{42}$ & Japón & II & GProx lap vs GT lap & 244 & cT1-2, cN0-1 & Morbilidad, mortalidad \\
\hline
\end{tabular}

GSTD = gastrectomía subtotal distal; lap = laparoscópica; $\mathrm{GT}$ = gastrectomía total; $\mathrm{SSN}$ = cirugía guiada con linfonodo centinela; $\mathrm{G}=$ gastrectomía; $\mathrm{PPG}=$ gastrectomía con preservación pilórica; GProx = gastrectomía proximal. 
gastrectomía para cáncer gástrico incipiente, pueden ser resumidas en los siguientes puntos, muchos de ellos controversiales: Omentectomía y bursectomía completa, disección linfática del hilio esplénico, manejo de borde esofágico en cáncer proximal, disección linfática $\mathrm{D} 2+$, resección multiorgánica y manejo de tumores de gran volumen ${ }^{44,45}$. Muchos de estos aspectos, considerados estándar o resueltos en cirugía abierta, han sido cuestionados y reanalizados; con el fin último de acomodar la evidencia a las dificultades técnicas de la cirugía laparoscópica.

Hasta el momento, han sido publicados una gran cantidad de estudios, de diverso grado de evidencia, que han intentado demostrar la seguridad y eficacia oncológica de la cirugía laparoscópica en tumores avanzados. Las primeras publicaciones de estudios randomizados, todas asiáticas, fueron principalmente esfuerzos individuales.

El primer estudio de este tipo fue publicado en el 2011, por Cai y cols. ${ }^{46}$. Fueron 123 pacientes en total, 61 de ellos sometidos a cirugía laparoscópica asistida. El grupo laparoscópico mostró un tiempo operatorio más prolongado que el grupo abierto, pero con menos pérdida sanguínea. Hubo menor frecuencia de complicaciones pulmonares en el grupo laparoscópico, pero la tasa total de morbilidad fue similar. En cuanto a los resultados a mediano plazo, no hubo diferencia estadísticamente significativa en la sobrevida global entre ambos grupos.

El segundo estudio fue publicado en el año 2016 por $\mathrm{Hu}^{47}$. Incluyó pacientes sometidos a gastrectomía distal laparoscópica (519) versus cirugía abierta (520). En ambos grupos la disección linfática estándar fue D2. El objetivo de la publicación fue demostrar que en manos de cirujanos con experiencia, la gastrectomía laparoscópica distal puede ser realizada en forma segura.

El tercer estudio randomizado, fue publicado en el año 2018 por Shi y cols. ${ }^{48}$. El objetivo de este trabajo era demostrar la no inferioridad de la cirugía laparoscópica sobre la abierta, en morbimortalidad operatoria. Se incluyeron 162 pacientes en el grupo laparoscópico y 160 pacientes en el grupo abierto. No hubo diferencias en morbilidad $(11,7 \%$ vs $14,4 \%$ ) y no hubo mortalidad en ambos grupos. Por lo tanto, la conclusión es que en centros experimentados, la gastrectomía laparoscópica para cáncer gástrico avanzado es segura y puede ser justificada en este grupo de pacientes.

Para agregar aún más evidencia que apoye el uso de la cirugía mínimamente invasiva en cáncer gástrico avanzado, en el año 2012 la Korean Laparoendoscopic Gastrointestinal Surgery Study (KLASS) lanzó un estudio multicéntrico, randomizado, pros- pectivo y fase III, cuyo objetivo fue comparar la eficacia de la cirugía laparoscópica, específicamente en la gastrectomía distal más linfadenectomía D2, en comparación con la cirugía convencional o abierta. El objetivo primario fue la sobrevida a tres años. Los objetivos secundarios fueron la morbilidad, mortalidad, índice de recuperación postoperatoria y la calidad de vida (KLASS-02) ${ }^{49,50}$. No contamos en la actualidad con resultados publicados a largo plazo. El único estudio randomizado que ha publicado sus resultados alejados (a 3 años) es el CLASS-0 $1^{51}$. En este estudio participaron 14 instituciones chinas, y sólo 15 cirujanos. Un hallazgo interesante es que en el grupo de pacientes con tumores pT4aN1$3 \mathrm{M} 0$, tuvieron mejor sobrevida los sometidos a gastrectomía distal abierta. La conclusión es que en tumores avanzados, en general, la gastrectomía distal laparoscópica no es inferior a la vía tradicional en cirujanos de gran experiencia.

El estudio japonés randomizado y multi-institucional JLSSG090152 evaluó 180 pacientes, divididos en grupos sometidos a gastrectomía subtotal distal abierta y laparoscópica, no encontrando diferencias significativas en resultados a corto plazo. Al igual que el KLASS-02, no están disponibles los resultados de eficacia a largo plazo.

Otros estudios multicéntricos, que evalúan la eficacia de la cirugía laparoscópica en cáncer gástrico avanzado (algunos evaluando el rol de la gastrectomía laparoscópica posterior a neoadyuvancia), están actualmente en curso (KLASS-03, JLSSG901, CLASS-01, LOGICA, COAT1001 y STOMACH) ${ }^{53-61}$. Se esperan resultados finales en los próximos años, los que nos darán mayores luces sobre la eficacia a largo plazo de la cirugía laparoscópica en cáncer gástrico avanzado (Tabla 2).

También han sido publicados múltiples metaanálisis que comparan la cirugía laparoscópica con la convencional en cáncer gástrico avanzado. Todos estos estudios demuestran que ambas técnicas tienen resultados similares en cuanto a morbimortalidad, cosecha linfonodal y tasas de recurrencia y sobrevida $^{65,66}$.

En resumen, existe suficiente evidencia de alto nivel para afirmar que en casos de cáncer gástrico incipiente, la gastrectomía subtotal laparoscópica es el estándar de tratamiento. La gastrectomía total para cáncer incipiente y avanzado, aún espera evidencia de estudios randomizados para definir su exacto rol. La gastrectomía subtotal para cáncer gástrico avanzado está apoyada en evidencia robusta, en relación a resultados postoperatorios precoces y alejados. Sin embargo, la falta de evidencia disponible no implica que esta vía de abordaje sea inferior a la 
Tabla 2. Principales estudios randomizados para cáncer gástrico avanzado: cirugía laparoscópica versus abierta

\begin{tabular}{|c|c|c|c|c|c|c|}
\hline Estudio & País & Fase & Intervención & $\begin{array}{l}\text { Muestra } \\
\text { pacientes }\end{array}$ & $\begin{array}{l}\text { Criterios } \\
\text { inclusión }\end{array}$ & “End point" \\
\hline KLASS $02^{49,50}$ & Corea & III & GSTD lap $v s$ abierta & 1.050 & cT2-4, cN0-1 & Sobrevida 3 años \\
\hline CLASSO $1^{51}$ & China & III & GSTD lap $v s$ abierta & 1.056 & cT2-4, cNo-3 & Sobrevida 3 años \\
\hline $\mathrm{ADDICT}^{61}$ & Corea & III & GSTD lap D1+ vs GSTD lap D2 & 1.880 & cT2-3, cN0-2 & Sobrevida 5 años \\
\hline KLASS06 ${ }^{63}$ & Corea & III & GT lap vs abierta & 772 & cT2-4, cN0-2 & Sobrevida 3 años \\
\hline Ziyu Li, Pekin ${ }^{60}$ & China & II & G lap vs G abierta & 180 & cT2-4a, cN0-3b & Morbilidad \\
\hline REALIZATION ${ }^{64}$ & China & III & GSTD lap (Neo) vs abierta (Neo) & 102 & $\begin{array}{c}\text { Neo: cT2-4a } \\
\text { cN+ M0 }\end{array}$ & Sobrevida 3 años \\
\hline
\end{tabular}

GSTD = gastrectomía subtotal distal; lap = laparoscópica; GT = gastrectomía total; SSN = cirugía guiada con linfonodo centinela; $\mathrm{G}=$ gastrectomía; $\mathrm{PPG}$ = gastrectomía con preservación pilórica; GProx = gastrectomía proximal; $\mathrm{Neo}=$ neoadyuvancia.

abierta y, por lo tanto, no debe llevar a falsas inferencias en la toma de decisiones de los grupos quirúrgicos más avezados en destreza laparoscópica ${ }^{38}$

\section{Responsabilidades éticas}

Protección de personas y animales: Los autores declaran que para esta investigación no se han realizado experimentos en seres humanos ni en animales.

Confidencialidad de los datos: Los autores declaran que han seguido los protocolos de su centro de trabajo sobre la publicación de datos de pacientes.

Derecho a la privacidad y consentimiento informado: Los autores declaran que en este artículo no aparecen datos de pacientes.

Conflicto de intereses: Los autores declaran no tener ningún conflicto de intereses.

\section{Bibliografía}

1. Thrift AP, El-Serag HB. Burden of Gastric Cancer. Clin Gastroenterol Hepatol. 2020;18:534-42. DOI: 10.1016/j. cgh.2019.07.045.

2. Rawla P, Barsouk A. Epidemiology of gastric cancer: global trends, risk factors and prevention. Prz Gastroenterol. 2019;14:26-38. DOI: $10.5114 /$ pg.2018.80001.

3. Balakrishnan M, George R, Sharma A, Graham DY. Changing Trends in Stomach Cancer Throughout the World.
Curr Gastroenterol Rep. 2017;19:36. DOI: $10.1007 / \mathrm{s} 11894-017-0575-8$.

4. García CC, Benavides CC, Apablaza PS, Rubilar OP, Covacevich RS, Peñaloza MP, et al. Resultados del tratamiento quirúrgico del cáncer gástrico: Análisis de 423 casos [Surgical treatment of gastric cancer: results in 423 cases]. Rev Med Chile 2007;135:687-95. DOI: 10.4067/S003498872007000600001

5. Mariette C, Carneiro F, Grabsch HI, Van der Post RS, Allum W, de Manzonni G. Consensus on the pathological definition and classification of poorly cohesive gastric carcinoma [published correction appears in Gastric Cancer 2019 Jan 10]. Gastric Cancer. 2019;22:1-9. DOI: $10.1007 / \mathrm{s} 10120-018-0868-0$.

6. Ang TL, Fock KM. Clinical epidemiology of gastric cancer. Singapore Med J. 2014;55:621-8. DOI: 10.11622/ smedj.2014174.

7. Karimi P, Islami F, Anandasabapathy S, Freedman ND, Kamangar F. Gastric cancer: descriptive epidemiology, risk factors, screening, and prevention. Cancer Epidemiol Biomarkers Prev. 2014;23:700- 


\section{DOI: 10.1158/1055-9965.EPI-13-} 1057.

8. Hamashima C. Current issues and future perspectives of gastric cancer screening. World J Gastroenterol. 2014;20:13767-74. DOI: $10.3748 /$ wjg.v20.i38.13767.

9. Huh YJ, Lee JH. The Advances of Laparoscopic Gastrectomy for Gastric Cancer. Gastroenterol Res Pract. 2017;2017:9278469. DOI: $10.1155 / 2017 / 9278469$.

10. Kitano S, Iso Y, Moriyama M, Sugimachi K. Laparoscopy-assisted Billroth I gastrectomy.Surg Laparosc Endosc. 1994;4:146-8.

11. Shiozaki H, Shimodaira Y, Elimova E, Wadhwa R, Sudo K, Harada K, et al. Evolution of gastric surgery techniques and outcomes. Chin J Cancer. 2016;35:69. Published 2016 Jul 26. DOI: 10.1186/ s40880-016-0134-y.

12. Zhao EH, Ling TL, Cao H. Current status of surgical treatment of gastric cancer in the era of minimally invasive surgery in China: Opportunity and challenge. Int J Surg. 2016;28:45-50. DOI: 10.1016/j. ijsu.2016.02.027.

13. Best LM, Mughal M, Gurusamy KS. Laparoscopic versus open gastrectomy for gastric cancer. Cochrane Database Syst Rev. 2016;3:CD011389. Published 2016 Mar 31. DOI: 10.1002/14651858. CD011389.pub2.

14. Etoh T, Shiroshita H, Shiraishi N, Kitano S, Inomata M. Ongoing clinical studies of minimally invasive surgery for gastric cancer in Japan. Transl Gastroenterol Hepatol. 2016;1:31. Published 2016 Apr 11. DOI: 10.21037/ tgh.2016.03.15.

15. Japanese Gastric Cancer Association. Japanese gastric cancer treatment guidelines 2014 (ver. 4). Gastric Cancer. 2017;20:1-19. DOI: 10.1007/s10120-0160622-4.

16. Japanese Gastric Cancer Association. Japanese gastric cancer treatment guidelines 2018 (5th edition) [published online ahead of print, $2020 \mathrm{Feb} 14]$. Gastric Cancer. 2020;10.1007/s10120020-01042-y. DOI: 10.1007/s10120-02001042-y.

17. Yamaguchi S, Sakata Y, Iwakiri R, Hara M, Akutagawa K, Shimoda R, et al. Increase in Endoscopic and Laparoscopic Surgery Regarding the Therapeutic
Approach of Gastric Cancer Detected by Cancer Screening in Saga Prefecture, Japan. Intern Med. 2016;55:1247-53. DOI: 10.2169/internalmedicine.55.5339.

18. Kitano S, Shiraishi N, Fujii K, Yasuda $\mathrm{K}$, Inomata $\mathrm{M}$, Adachi $\mathrm{Y}$. A randomized controlled trial comparing open $v$ s laparoscopy-assisted distal gastrectomy for the treatment of early gastric cancer: an interim report. Surgery 2002;131(1 Suppl):S306-S311. DOI: 10.1067/ msy.2002.120115.

19. Kim W, Kim HH, Han SU, Kim MC, Hyung WJ, Ryu SW, et al. Decreased Morbidity of Laparoscopic Distal Gastrectomy Compared With Open Distal Gastrectomy for Stage I Gastric Cancer: Short-term Outcomes From a Multicenter Randomized Controlled Trial (KLASS-01). Ann Surg. 2016;263:28-35. DOI: $10.1097 /$ SLA.0000000000001346.

20. Kim HH, Han SU, Kim MC, Kim W, Lee HJ, Ryu SW, et al. Effect of Laparoscopic Distal Gastrectomy vs Open Distal Gastrectomy on Longterm Survival Among Patients With Stage I Gastric Cancer: The KLASS-01 Randomized Clinical Trial. JAMA Oncol. 2019;5:506-13. DOI: 10.1001/ jamaoncol.2018.6727.

21. Katai H, Mizusawa J, Katayama H, Takagi M, Yoshikawa T, Fukagawa T, et al. Shortterm surgical outcomes from a phase III study of laparoscopy-assisted versus open distal gastrectomy with nodal dissection for clinical stage IA/IB gastric cancer: Japan Clinical Oncology Group Study JCOG0912. Gastric Cancer 2017;20:699708. DOI: 10.1007/s10120-016-0646-9.

22. Katai H, Mizusawa J, Katayama H, Morita S, Yamada T, Bando E, et al. Survival outcomes after laparoscopyassisted distal gastrectomy versus open distal gastrectomy with nodal dissection for clinical stage IA or IB gastric cancer (JCOG0912): a multicentre, noninferiority, phase 3 randomised controlled trial. Lancet Gastroenterol Hepatol. 2020;5:142-51. DOI: 10.1016/S24681253(19)30332-2.

23. Kim YW, Yoon HM, Yun YH, Nam BH, Eom BW, Baik YH, et al. Long-term outcomes of laparoscopy-assisted distal gastrectomy for early gastric cancer: result of a randomized controlled trial (COACT 0301). Surg Endosc. 2013;27:4267-76.
DOI: 10.1007/s00464-013-3037-x.

24. Best LM, Mughal M, Gurusamy KS. Laparoscopic versus open gastrectomy for gastric cancer. Cochrane Database Syst Rev. 2016;3:CD011389. Published 2016 Mar 31. DOI: 10.1002/14651858. CD011389.pub2.

25. Pan Y, Chen K, Yu WH, Maher H, Wang S, Zhao H, et al. Laparoscopic gastrectomy for elderly patients with gastric cancer: A systematic review with meta-analysis. Medicine (Baltimore). 2018;97:e0007. DOI: 10.1097/ MD.0000000000010007.

26. Zhang CD, Yamashita H, Zhang S, Seto Y. Reevaluation of laparoscopic versus open distal gastrectomy for early gastric cancer in Asia: A meta-analysis of randomized controlled trials. Int J Surg. 2018;56:3143. DOI: 10.1016/j.ijsu.2018.05.733.

27. Wei Y, Yu D, Li Y, Fan C, Li G. Laparoscopic versus open gastrectomy for advanced gastric cancer: A meta-analysis based on high-quality retrospective studies and clinical randomized trials. Clin Res Hepatol Gastroenterol. 2018;42:577-90. DOI: 10.1016/j.clinre.2018.04.005.

28. Berlth F, Yang HK. Minimal-invasive gastrectomy: what the west can learn from the east?. Updates Surg. 2018;70:181-7. DOI: 10.1007/s13304-018-0547-z.

29. Özer İ, Bostancı EB, Ulaş M, Özoğul Y, Akoğlu M. Changing Trends in Gastric Cancer Surgery. Balkan Med J. 2017;34:10-20. DOI: 10.4274/ balkanmedj.2015.1461.

30. Adachi Y, Kitano S, Sugimachi K. Surgery for gastric cancer: 10-year experience worldwide. Gastric Cancer. 2001;4:16674. DOI: 10.1007/s10120-001-8007-7.

31. Sajid MS, Hebbar M, Sayegh ME. Minimally invasive surgery for gastric cancer in UK: current status and future perspectives. Transl Gastroenterol Hepatol. 2017;2:44. Published 2017 May 9. DOI: $10.21037 / \operatorname{tgh} .2017 .04 .06$.

32. Strong VE, Devaud N, Karpeh M. The role of laparoscopy for gastric surgery in the West. Gastric Cancer. 2009;12:127-31. DOI: 10.1007/s10120-008-0516-1.

33. Strong VE. Laparoscopic resection for gastric carcinoma: Western experience. Surg Oncol Clin N Am. 2012;21:141-58. DOI: 10.1016/j.soc.2011.09.010.

34. Huscher CG, Mingoli A, Sgarzini G, Sansonetti A, Di Paola M, Recher A, et 
al. Laparoscopic versus open subtotal gastrectomy for distal gastric cancer: fiveyear results of a randomized prospective trial. Ann Surg. 2005;241:232-7. DOI: 10.1097/01.sla.0000151892.35922. f2.

35. He H, Li H, Su X, Li Z, Yu P, Sun Y, et al. Study on safety of laparoscopic total gastrectomy for clinical stage I gastric cancer: the protocol of the CLASS02-01 multicenter randomized controlled clinical trial. BMC Cancer. 2018;18:944. Published 2018 Oct 3. DOI: 10.1186/ s12885-018-4846-z.

36. Hyung WJ, Yang HK, Han SU, Lee YJ, Park JM, Kim JJ, et al. A feasibility study of laparoscopic total gastrectomy for clinical stage I gastric cancer: a prospective multi-center phase II clinical trial, KLASS 03. Gastric Cancer 2019;22:214-22. DOI: 10.1007/s10120018-0864-4.

37. Xiong JJ, Nunes QM, Huang W, Tan CL, Ke NW, Xie SM, et al. Laparoscopic vs open total gastrectomy for gastric cancer: a meta-analysis. World J Gastroenterol. 2013;19:8114-32. DOI: 10.3748/wjg.v19. i44.8114.

38. Braithwaite RS. A piece of my mind. EBM's six dangerous words. JAMA. 2013;310:2149-50. DOI: 10.1001/ jama.2013.281996.

39. An JY, Min JS, Lee YJ, Jeong SH, Hur H, Han SU, et al. Safety of Laparoscopic Sentinel Basin Dissection in Patients with Gastric Cancer: an Analysis from the SENORITA Prospective Multicenter Quality Control Trial. J Gastric Cancer 2018;18:30-6. DOI: 10.5230/jgc.2018.18. e6.

40. Oh SY, Lee HJ, Yang HK. PylorusPreserving Gastrectomy for Gastric Cancer. J Gastric Cancer. 2016;16:63-71. DOI: 10.5230/jgc.2016.16.2.63.

41. Park DJ, Park YS, Ahn SH, Kim HH. Laparoscopic Proximal Gastrectomy as a Surgical Treatment for Upper Third Early Gastric Cancer. Korean J Gastroenterol. 2017;70:134-140. DOI: 10.4166/ kjg.2017.70.3.134.

42. Katai H, Mizusawa J, Katayama H, Kunisaki C, Sakuramoto S, Inaki N, et al. Single-arm confirmatory trial of laparoscopy-assisted total or proximal gastrectomy with nodal dissection for clinical stage I gastric cancer:
Japan Clinical Oncology Group study JCOG1401. Gastric Cancer. 2019;22:999-1008. DOI: 10.1007/s10120019-00929-9.

43. Jin HE, Kim MS, Lee CM, Park JH, Choi CI, Lee HH, et al. Meta-analysis and systematic review on laparoscopicassisted distal gastrectomy (LADG) and totally laparoscopic distal gastrectomy (TLDG) for gastric cancer: Preliminary study for a multicenter prospective KLASS07 trial. Eur J Surg Oncol. 2019;45:2231-2240. DOI: 10.1016/j. ejso.2019.06.030.

44. Antonakis PT, Ashrafian H, Isla AM. Laparoscopic gastric surgery for cancer: where do we stand?. World J Gastroenterol. 2014;20:14280-91. DOI: $10.3748 /$ wjg.v20.i39.14280.

45. Lianos GD, Hasemaki N, Glantzounis GK, Mitsis M, Rausei S. Assessing safety and feasibility of 'pure' laparoscopic total gastrectomy for advanced gastric cancer in the West. Review article. Int J Surg. 2018;53:275-8. DOI: 10.1016/j. ijsu.2018.03.048.

46. Cai J, Wei D, Gao CF, Zhang CS, Zhang $\mathrm{H}$, Zhao T. A prospective randomized study comparing open versus laparoscopyassisted D2 radical gastrectomy in advanced gastric cancer. Dig Surg. 2011;28:331-7. DOI: 10.1159/000330782.

47. Hu Y, Huang C, Sun Y, Su X, Cao H, $\mathrm{Hu}$ J, et al. Morbidity and Mortality of Laparoscopic versus Open D2 Distal Gastrectomy for Advanced Gastric Cancer: A Randomized Controlled Trial. J Clin Oncol. 2016;34:1350-7. DOI: 10.1200/JCO.2015.63.7215.

48. Shi Y, Xu X, Zhao Y, Qian F, Tang B, Hao $Y$, et al. Short-term surgical outcomes of a randomized controlled trial comparing laparoscopic versus open gastrectomy with D2 lymph node dissection for advanced gastric cancer. Surg Endosc. 2018;32:2427-33. DOI: 10.1007/s00464017-5942-x.

49. Hur H, Lee HY, Lee HJ, Kim MC, Hyung WJ, Park YK, et al. Efficacy of laparoscopic subtotal gastrectomy with D2 lymphadenectomy for locally advanced gastric cancer: the protocol of the KLASS-02 multicenter randomized controlled clinical trial. BMC Cancer 2015;15:355. Published 2015 May 5. DOI: 10.1186/s12885-015-1365-z.
50. Lee HJ, Hyung WJ, Yang HK, Han SU, Park YK, An JY, et al. Shortterm Outcomes of a Multicenter Randomized Controlled Trial Comparing Laparoscopic Distal Gastrectomy With D2 Lymphadenectomy to Open Distal Gastrectomy for Locally Advanced Gastric Cancer (KLASS-02-RCT). Ann Surg. 2019;270:983-91. DOI: 10.1097/ SLA.0000000000003217.

51. Yu J, Huang C, Sun Y, Su X, Cao H, $\mathrm{Hu}$ J, et al. Effect of Laparoscopic vs Open Distal Gastrectomy on 3-Year Disease-Free Survival in Patients With Locally Advanced Gastric Cancer: The CLASS-01 Randomized Clinical Trial. JAMA. 2019;321:1983-92. DOI: 10.1001/ jama.2019.5359.

52. Inaki $\mathrm{N}$, Etoh $\mathrm{T}$, Ohyama $\mathrm{T}$, Uchiyama K, Katada N, Koeda K, et al. A Multiinstitutional, Prospective, Phase II Feasibility Study of Laparoscopy-Assisted Distal Gastrectomy with D2 Lymph Node Dissection for Locally Advanced Gastric Cancer (JLSSG0901). World J Surg. 2015;39:2734-41. DOI: 10.1007/s00268015-3160-z.

53. Yang HK, Hyung WJ, Han SU, Lee YJ, Park JM, Cho GS, et al. Comparison of surgical outcomes among different methods of esophagojejunostomy in laparoscopic total gastrectomy for clinical stage I proximal gastric cancer: results of a single-arm multicenter phase II clinical trial in Korea, KLASS 03 [published online ahead of print, 2020 Mar 6]. Surg Endosc. 2020;10.1007/s00464-020-074800. DOI: $10.1007 / \mathrm{s} 00464-020-07480-0$.

54. Hyung WJ, Yang HK, Han SU, Lee YJ, Park JM, Kim JJ, et al. A feasibility study of laparoscopic total gastrectomy for clinical stage I gastric cancer: a prospective multi-center phase II clinical trial, KLASS 03. Gastric Cancer. 2019;22:214-22. DOI: 10.1007/s10120018-0864-4.

55. Yu J, Huang C, Sun Y, Su X, Cao H, $\mathrm{Hu}$ J, et al. Effect of Laparoscopic vs Open Distal Gastrectomy on 3-Year Disease-Free Survival in Patients With Locally Advanced Gastric Cancer: The CLASS-01 Randomized Clinical Trial. JAMA. 2019;321:1983-92. DOI: 10.1001/ jama.2019.5359.

56. Park YK, Yoon HM, Kim YW, Park JY, Ryu KW, Lee YJ, et al. 
Laparoscopy-assisted versus Open D2 Distal Gastrectomy for Advanced Gastric Cancer: Results From a Randomized Phase II Multicenter Clinical Trial (COACT 1001). Ann Surg. 2018;267:638-45. DOI: 10.1097/ SLA.0000000000002168.

57. Byun C, Han SU. Current status of randomized controlled trials for laparoscopic gastric surgery for gastric cancer in Korea. Asian J Endosc Surg. 2015;8:130-8. DOI: 10.1111/ases.12176.

58. Hu J, Zhang W, Chen X. Laparoscopic Gastrectomy Combined With Neoadjuvant Chemotherapy for Gastric Cancer Patients: From the View of the CLASS03a Trial. Zhonghua Wei Chang Wai Ke Za Zhi. 2018;21:138-42.

59. Straatman J, van der Wielen N, Cuesta MA, Gisbertz SS, Hartemink KJ, Poza AA, et al. Surgical techniques, open versus minimally invasive gastrectomy after chemotherapy (STOMACH trial): study protocol for a randomized controlled trial. Trials 2015;16:123. Published 2015 Mar 27. DOI: 10.1186/s13063-015-06389.
60. Li Z, Shan F, Ying X, Zhang L, Ren $\mathrm{H}$, Lii S, et al. Laparoscopic or open distal gastrectomy after neoadjuvant chemotherapy for advanced gastric cancer: study protocol for a randomised phase II trial. BMJ Open. 2018;8:e021633. Published 2018 Aug 10. DOI: 10.1136/ bmjopen-2018-021633.

61. Kim J, Eom BW, Nam BH, Yoon HM, Kim YW. ADDICT - A multicenter randomized clinical trial of $\mathrm{D} 1+$ versus D2 distal gastrectomy for stage IB \& II advanced gastric cancer: Design and rationale. EJSO. 2014; 40: S162. DOI: 10.1016/j.ejso.2014.08.413.

62. Haverkamp L, Brenkman HJ, Seesing MF, Gisbertz SS, van Berge Henegouwen MI, Luyer MD, et al. Laparoscopic versus open gastrectomy for gastric cancer, a multicenter prospectively randomized controlled trial (LOGICA-trial). BMC Cancer. 2015;15:556. Published 2015 Jul 29. DOI: $10.1186 / \mathrm{s} 12885-015-1551-\mathrm{z}$.

63. Zhu C, Zhao G, Cao H. Clinical Trials of Laparoscopic Gastric Cancer Surgery in South Korea: Review and Prospect. Zhonghua Wei Chang Wai
Ke Za Zhi. 2018;21:154-9. Published 2018 Feb 25. DOI: $10.3760 / \mathrm{cma}$.j.is sn.1671-0274.2018.02.007.

64. Li Z, Shan F, Ying X, Zhang Y, E JY, Wang Y, et al. Assessment of Laparoscopic Distal Gastrectomy After Neoadjuvant Chemotherapy for Locally Advanced Gastric Cancer: A Randomized Clinical Trial. JAMA Surg. 2019;154:1093-1101. DOI: 10.1001/ jamasurg.2019.3473.

65. Li Z, Zhao Y, Lian B, Liu Y, Zhao Q. Long-term oncological outcomes in laparoscopic versus open gastrectomy for advanced gastric cancer: A metaanalysis of high-quality nonrandomized studies. Am J Surg. 2019;218:631-8. DOI: 10.1016/j.amjsurg.2019.01.020.

66. Beyer K, Baukloh AK, Kamphues C, Seelinger H, Heidecke CD, Kreis ME, et al. Laparoscopic versus open gastrectomy for locally advanced gastric cancer: a systematic review and meta-analysis of randomized controlled studies. World J Surg Oncol. 2019;17:68. Published 2019 Apr 15. DOI: 10.1186/s12957-019-16001. 